\title{
A Note on Surface Integrals of Vector Fields
}

\author{
Zhengyuan Wei, Xiaoya Zhou, Jinrong Jiang \\ College of Science, Chongqing University of Technology, Chongqing, China \\ Email: weizy@cqut.edu.cn,xyzhou@2019.cqut.edu.cn,jjr@2020.cqut.edu.cn
}

How to cite this paper: Wei, Z.Y., Zhou, X.Y. and Jiang, J.R. (2021) A Note on Surface Integrals of Vector Fields. Open Access Library Journal, 8: e7934. https://doi.org/10.4236/oalib.1107934

Received: September 7, 2021

Accepted: September 27, 2021

Published: September 30, 2021

Copyright $\odot 2021$ by author(s) and Open Access Library Inc.

This work is licensed under the Creative Commons Attribution International License (CC BY 4.0).

http://creativecommons.org/licenses/by/4.0/ (c) (i)

Open Access

\begin{abstract}
Surface integrals of vector fields play an important role in the solutions of natural science and physical science. The Gauss theorem reduces the difficulty of directly computing surface integrals of vector fields. This paper introduces an approach for the computation of integral surfaces in vector fields and obtains a generalized mathematical expression based on Gauss theorem. Moreover, the computation time is investigated by two classical examples.
\end{abstract}

\section{Subject Areas}

Applied Physics, Financial Mathematics, Mathematical Analysis, Mathematical Statistics, Mathematics

\section{Keywords}

Surface Integrals, Gauss Theorem, Green Theorem

\section{Introduction}

The direct computation of surface integrals of vector fields [1] is quite difficult, but we can simplify the derivation of the result using the Gauss theorem [2] [3]. However, the Gauss theorem can be used to calculate a flux through a closed surface that fully encloses a volume, but it can not directly be used to calculate the flux through surfaces with boundaries. See examples below:

Example 1: Evaluate $\oiint_{\Sigma} \frac{x \mathrm{~d} y \mathrm{~d} z+y \mathrm{~d} z \mathrm{~d} x+z \mathrm{~d} x \mathrm{~d} y}{\left(x^{2}+y^{2}+z^{2}\right)^{\frac{3}{2}}}$, where $\Sigma$ is the lateral surface of $2 x^{2}+2 y^{2}+z^{2}=4$.

Example 2: Evaluate $\iint_{\Sigma} \frac{x \mathrm{~d} y \mathrm{~d} z+y \mathrm{~d} z \mathrm{~d} x+z \mathrm{~d} x \mathrm{~d} y}{\left(x^{2}+y^{2}+z^{2}\right)^{\frac{3}{2}}}$, where $\Sigma$ is the lateral surface of $1-\frac{z}{5}=\frac{(x-2)^{2}}{16}+\frac{(y-1)^{2}}{9}, z>0$. 
The above example doesn't satisfy the condition that the integrand has a first-order continuous partial derivative in the closed space, and Gauss theorem cannot be used directly. In order to use the Gauss theorem in the flux through surfaces with boundaries, we often construct a closed surface that fully encloses a volume through a complemented surface in the boundaries.

In this paper, we first discuss a classical surface integral of vector fields, then introduce an approach for the computation of integral surfaces in vector fields and obtain a generalized mathematical expression based on Gauss theorem. It turns out that with these conclusions the computation of common complex integral, such as Example 1 and Example 2, within minimum time is realized. The integral given by theorem 1 is more general. The research on surface integral of vector field in this paper will enrich and develop the integral theory to a certain extent, and provide reference for other application fields.

\section{Main Results}

Lemma 1: 1) Suppose $\Omega$ is a subset of $R^{3}$ which is compact and has a piecewise smooth boundary $\Sigma ;(0,0,0) \in \Omega ; f(x, y, z), g(x, y, z), h(x, y, z)$ are continuously differentiable vector field defined on a neighborhood of $\Omega ; 2$ ) Let:

$\left(\frac{\partial f}{\partial x}+\frac{\partial g}{\partial y}+\frac{\partial h}{\partial z}\right)\left(a x^{2}+b y^{2}+c z^{2}\right)=n(a x f+b y g+c z h)$, for any $a>0, b>0, c>0$ and $n \in N_{+}$. Then we have:

$$
\begin{aligned}
& \oiint_{\Sigma} \frac{f(x, y, z) \mathrm{d} y \mathrm{~d} z+g(x, y, z) \mathrm{d} z \mathrm{~d} x+h(x, y, z) \mathrm{d} x \mathrm{~d} y}{\left(a x^{2}+b y^{2}+c z^{2}\right)^{\frac{n}{2}}} \\
& =\frac{1}{\delta^{n}} \iiint_{a x^{2}+b y^{2}+c z^{2} \leq \delta^{2}}\left(\frac{\partial f}{\partial x}+\frac{\partial g}{\partial y}+\frac{\partial h}{\partial z}\right) \mathrm{d} v
\end{aligned}
$$

Remark 1 (1) Take:

$n=3, f(x, y, z)=x, g(x, y, z)=y, h(x, y, z)=z, a=1, b=1, c=1$. It is intuitively clear from the construction of the integral that the example 1 and example 2 belong to the Equation (1). A general application of the Equation (1) is in natural science and physical science.

Theorem 1: Suppose $\Omega$ is a subset of $R^{3}$ which is compact and has a piecewise smooth boundary $\Sigma ;(0,0,0) \in \Omega ; f(x, y, z), g(x, y, z), h(x, y, z)$ are continuously differentiable vector field defined on a neighborhood of $\Omega$; $a>0, b>0, c>0$. Then:

$$
\iint_{\Sigma} \frac{x \mathrm{~d} y \mathrm{~d} z+y \mathrm{~d} z \mathrm{~d} x+z \mathrm{~d} x \mathrm{~d} y}{\left(a x^{2}+b y^{2}+c z^{2}\right)^{\frac{3}{2}}}=\frac{4 \pi}{\sqrt{a b c}}
$$

1) Let:

$$
P(x, y, z)=\frac{x}{\left(a x^{2}+b y^{2}+c z^{2}\right)^{\frac{3}{2}}}
$$




$$
\begin{aligned}
& Q(x, y, z)=\frac{y}{\left(a x^{2}+b y^{2}+c z^{2}\right)^{\frac{3}{2}}} \\
& R(x, y, z)=\frac{z}{\left(a x^{2}+b y^{2}+c z^{2}\right)^{\frac{3}{2}}}
\end{aligned}
$$

Denote by $\Sigma_{1}$, a small surface taken within the integral surfaces. The space bounded by $\Sigma_{1}$ is denoted by $\Omega_{1}$. The space bounded by $\Sigma_{1}$ and $\Sigma$ is denoted by $\Omega_{2}$, satisfying $\Omega=\Omega_{1} \cup \Omega_{2},(0,0,0) \in \Omega_{1}$.

2) It is easy to know that the partial derivatives of functions $P, Q, R$ are continuous on the place $\Omega_{2}$ and $\frac{\partial P}{\partial x}+\frac{\partial Q}{\partial y}+\frac{\partial R}{\partial z}=0$

3) Using Gauss theorem, we have:

$$
\begin{aligned}
& \oiint_{\Sigma} P \mathrm{~d} y \mathrm{~d} z+Q \mathrm{~d} z \mathrm{~d} x+R \mathrm{~d} z \mathrm{~d} y \\
& =\oiint_{\Sigma+\Sigma_{1}^{-}} P \mathrm{~d} y \mathrm{~d} z+Q \mathrm{~d} z \mathrm{~d} x+R \mathrm{~d} z \mathrm{~d} y-\oiint_{\Sigma} P \mathrm{~d} y \mathrm{~d} z+Q \mathrm{~d} z \mathrm{~d} x+R \mathrm{~d} z \mathrm{~d} y \\
& =\iiint \int_{\Omega_{2}}\left(\frac{\partial P}{\partial x}+\frac{\partial Q}{\partial y}+\frac{\partial R}{\partial z}\right) \mathrm{d} x \mathrm{~d} y \mathrm{~d} z-\oiint_{\Sigma_{1}^{-}} P \mathrm{~d} y \mathrm{~d} z+Q \mathrm{~d} z \mathrm{~d} x+R \mathrm{~d} z \mathrm{~d} y \\
& =\oiint_{\Sigma_{1}} \frac{x \mathrm{~d} y \mathrm{~d} z+y \mathrm{~d} z \mathrm{~d} x+z \mathrm{~d} x \mathrm{~d} y}{\left(a x^{2}+b y^{2}+c z^{2}\right)^{\frac{3}{2}}}
\end{aligned}
$$

where $\Sigma_{1}$ is the outer edge of the surface, $\Sigma_{1}^{-}$is the inner side of the surface.

4) The speed of solving surface integrals of vector fields depends on the surface shape that we take. By introducing a surface $\Sigma_{1}$, solutions to the Equation (2) are given by the solutions to the other integral equations. Two kinds of methods has be shown in the following:

a) Take $\Sigma_{1}$ as a small oval surface $\left(a x^{2}+b y^{2}+c z^{2} \leq \delta^{2}\right)$, see Figure 1 .

so the Equation (2) can be written in the form:

$$
\oiint_{\Sigma_{1}} \frac{x \mathrm{~d} y \mathrm{~d} z+y \mathrm{~d} z \mathrm{~d} x+z \mathrm{~d} x \mathrm{~d} y}{\left(a x^{2}+b y^{2}+c z^{2}\right)^{\frac{3}{2}}}=\frac{1}{\delta^{3}} \oiint_{\Sigma_{1}} x \mathrm{~d} y \mathrm{~d} z+y \mathrm{~d} z \mathrm{~d} x+z \mathrm{~d} x \mathrm{~d} y
$$

Calculation of Equation (3) is often done by using the following methods:

i) Using Gauss theorem [2],

Indeed:

$$
\begin{aligned}
& \oiint_{\Sigma_{1}} x \mathrm{~d} y \mathrm{~d} z+y \mathrm{~d} z \mathrm{~d} x+z \mathrm{~d} x \mathrm{~d} y=3 \iiint_{\Omega} \mathrm{d} x \mathrm{~d} y \mathrm{~d} z \\
& =3 \times \frac{4 \pi}{3} \times \frac{\delta}{\sqrt{a}} \times \frac{\delta}{\sqrt{b}} \times \frac{\delta}{\sqrt{c}}=\frac{4 \pi \delta^{3}}{\sqrt{a b c}}
\end{aligned}
$$

then we obtain:

$$
\oiint_{\Sigma_{1}} \frac{x \mathrm{~d} y \mathrm{~d} z+y \mathrm{~d} z \mathrm{~d} x+z \mathrm{~d} x \mathrm{~d} y}{\left(a x^{2}+b y^{2}+c z^{2}\right)^{\frac{3}{2}}}=\frac{4 \pi}{\sqrt{a b c}}
$$

ii) Using the connection of two kinds of surface integral. 


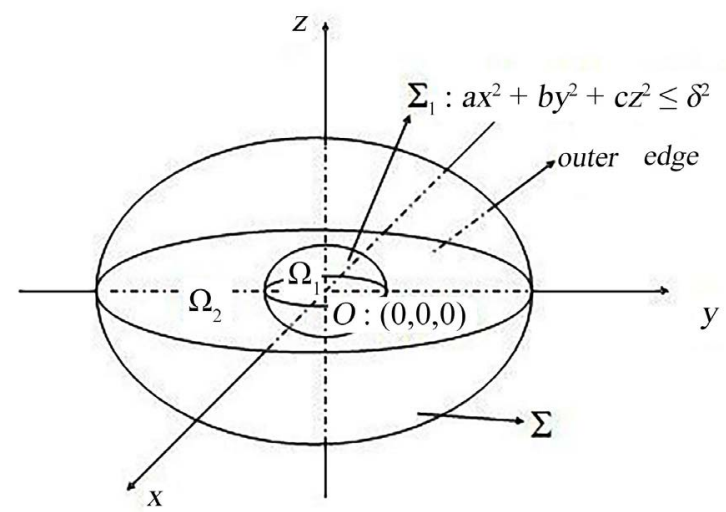

Figure 1. Graphs of $\Sigma$ and $\Sigma_{1}$.

Project the surface on the xoy plane, see Figure 2.

Let:

$$
\begin{aligned}
& \Sigma_{1(\text { above })}: z=\frac{1}{\sqrt{c}} \sqrt{\delta^{2}-a x^{2}-b y^{2}} \\
& \Sigma_{1(\text { below })}: z=\frac{-1}{\sqrt{c}} \sqrt{\delta^{2}-a x^{2}-b y^{2}}
\end{aligned}
$$

Then:

$$
\begin{aligned}
& \oiint_{\Sigma_{1}} x \mathrm{~d} y \mathrm{~d} z+y \mathrm{~d} z \mathrm{~d} x+z \mathrm{~d} x \mathrm{~d} y \\
& =\oiint_{\Sigma_{1(a b o v e)}} x \mathrm{~d} y \mathrm{~d} z+y \mathrm{~d} z \mathrm{~d} x+z \mathrm{~d} x \mathrm{~d} y+\oiint_{\Sigma_{1(\text { below })}} x \mathrm{~d} y \mathrm{~d} z+y \mathrm{~d} z \mathrm{~d} x+z \mathrm{~d} x \mathrm{~d} y \\
& \left.=2 \oiint_{\Sigma_{1(\text { above })}} x \cdot\left(-\frac{\partial z}{\partial x}\right)+y \cdot\left(-\frac{\partial z}{\partial y}\right)+z\right] \mathrm{d} x \mathrm{~d} y \\
& =\frac{2 \delta^{2}}{\sqrt{c}} \oiint_{\left.\Sigma_{1(\text { above }}\right)} \frac{1}{\sqrt{\delta^{2}-a x^{2}-b y^{2}}} \mathrm{~d} x \mathrm{~d} y
\end{aligned}
$$

Using polar coordinate transformation. If $x, y$ are transformed into $r, \theta$ by a transformation $\left\{\begin{array}{l}x=\frac{1}{\sqrt{a}} r \cos \theta \\ y=\frac{1}{\sqrt{b}} r \sin \theta\end{array}\right.$, then we have Equation (4) as follows:

$$
\begin{aligned}
& \frac{2 \delta^{2}}{\sqrt{c}} \oiint_{\Sigma_{1}(\text { above })} \frac{1}{\sqrt{\delta^{2}-a x^{2}-b y^{2}}} \mathrm{~d} x \mathrm{~d} y \\
& =\frac{2 \delta^{2}}{\sqrt{c}} \int_{0}^{2 \pi} \mathrm{d} \theta \int_{0}^{\delta} \frac{1}{\sqrt{\delta^{2}-r^{2}}} \cdot \frac{1}{\sqrt{a}} \cdot \frac{1}{\sqrt{b}} r \mathrm{~d} r \\
& =\frac{4 \pi \delta^{2}}{\sqrt{a b c}} \int_{0}^{\delta} \frac{r}{\sqrt{\delta^{2}-r^{2}}} \mathrm{~d} r \\
& =\frac{4 \pi \delta^{3}}{\sqrt{a b c}}
\end{aligned}
$$

Then we obtain: 


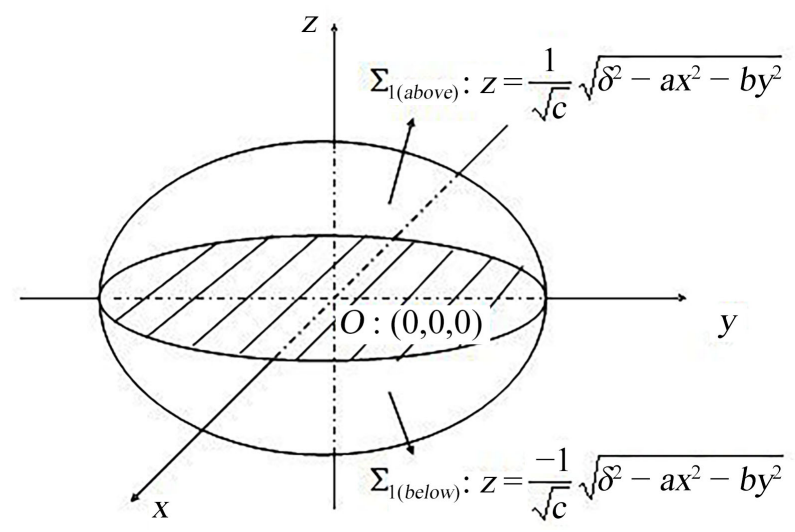

Figure 2. Graphs of $\Sigma_{1}$.

$$
\oiint_{\Sigma_{1}} \frac{x \mathrm{~d} y \mathrm{~d} z+y \mathrm{~d} z \mathrm{~d} x+z \mathrm{~d} x \mathrm{~d} y}{\left(a x^{2}+b y^{2}+c z^{2}\right)^{\frac{3}{2}}}=\frac{4 \pi}{\sqrt{a b c}}
$$

iii) Using the relationship between the two kinds of surface integrals.

$$
\begin{aligned}
& \oiint_{\Sigma_{1}} x \mathrm{~d} y \mathrm{~d} z+y \mathrm{~d} z \mathrm{~d} x+z \mathrm{~d} x \mathrm{~d} y \\
& =\oiint_{\Sigma_{1(\text { above })}: z=\frac{1}{\sqrt{c}} \sqrt{\delta^{2}-a x^{2}-b y^{2}}}(x \cos \alpha+y \cos \beta+z \cos \gamma) \mathrm{d} S \\
& +\oiint_{\Sigma_{1(\text { below }): z}=\frac{-1}{\sqrt{c}} \sqrt{\delta^{2}-a x^{2}-b y^{2}}}(x \cos \alpha+y \cos \beta+z \cos \gamma) \mathrm{d} S
\end{aligned}
$$

where on surface $\Sigma_{1(\text { above })}$, we have:

$$
\begin{aligned}
(\cos \alpha, \cos \beta, \cos \gamma)= & \left(\frac{-z_{x}^{\prime}}{\sqrt{1+z_{x}^{\prime 2}+z_{y}^{\prime 2}}}, \frac{-z_{y}^{\prime}}{\sqrt{1+z_{x}^{\prime 2}+z_{y}^{\prime 2}}}, \frac{1}{\sqrt{1+z_{x}^{\prime 2}+z_{y}^{\prime 2}}}\right) \\
a x^{2}+b y^{2}+c z^{2} & =\delta^{2}, z>0
\end{aligned}
$$

Let:

$$
F(x, y, z)=a x^{2}+b y^{2}+c z^{2}-\delta^{2}
$$

then we have:

$$
\begin{gathered}
\frac{\partial F}{\partial x}=2 a x, \frac{\partial F}{\partial y}=2 b y, \frac{\partial F}{\partial z}=2 c z \\
z_{x}^{\prime}=-\frac{\partial F / \partial x}{\partial F / \partial z}=-\frac{a x}{c z}, z_{y}^{\prime}=-\frac{\partial F / \partial y}{\partial F / \partial z}=-\frac{b y}{c z} \\
\sqrt{1+z_{x}^{\prime 2}+z_{y}^{\prime 2}}=\frac{\sqrt{a^{2} x^{2}+b^{2} y^{2}+c^{2} z^{2}}}{c z} \\
\cos \alpha=-\frac{z_{x}^{\prime}}{\sqrt{1+z_{x}^{\prime 2}+z_{y}^{\prime 2}}}=\frac{a x}{\sqrt{a^{2} x^{2}+b^{2} y^{2}+c^{2} z^{2}}} \\
\cos \beta=-\frac{z_{y}^{\prime}}{\sqrt{1+z_{x}^{\prime 2}+z_{y}^{\prime 2}}}=\frac{b y}{\sqrt{a^{2} x^{2}+b^{2} y^{2}+c^{2} z^{2}}}
\end{gathered}
$$




$$
\cos \gamma=\frac{1}{1+z_{x}^{\prime 2}+z_{y}^{\prime 2}}=\frac{c z}{\sqrt{a^{2} x^{2}+b^{2} y^{2}+c^{2} z^{2}}}
$$

That is:

$$
(\cos \alpha, \cos \beta, \cos \gamma)=\frac{1}{\sqrt{a^{2} x^{2}+b^{2} y^{2}+c^{2} z^{2}}}(a x, b y, c z)
$$

Similarly on surface $\Sigma_{1(\text { below })}$, we have:

$$
(\cos \alpha, \cos \beta, \cos \gamma)=-\frac{1}{\sqrt{a^{2} x^{2}+b^{2} y^{2}+c^{2} z^{2}}}(a x, b y, c z)
$$

since the surface of $a x^{2}+b y^{2}+c z^{2}=\delta^{2}$ is symmetric about the xoy plane, we have:

$$
\begin{aligned}
& \oiint_{\Sigma_{1(b e l o w)}: z=\frac{-1}{\sqrt{c}} \sqrt{\delta^{2}-a x^{2}-b y^{2}}}(x \cos \alpha+y \cos \beta+z \cos \gamma) \mathrm{d} S \\
& =\oiint_{\Sigma_{1(\text { below }): z=\frac{-1}{\sqrt{c}} \sqrt{\delta^{2}-a x^{2}-b y^{2}}}}\left(-\frac{a x^{2}+b y^{2}+c z^{2}}{\sqrt{a^{2} x^{2}+b^{2} y^{2}+c^{2} z^{2}}}\right) \mathrm{d} S \\
& =\delta^{2} \oiint_{\Sigma_{1(b e l o w)}: a x^{2}+b y^{2}<\delta^{2}}\left(\frac{\sqrt{1+z_{x}^{\prime 2}+z_{y}^{\prime 2}}}{\sqrt{a^{2} x^{2}+b^{2} y^{2}+c^{2} z^{2}}}\right) \mathrm{d} x \mathrm{~d} y \\
& =\frac{\delta^{2}}{\sqrt{c}} \oiint_{\Sigma_{1(b e l o w)} a x^{2}+b y^{2}<\delta^{2}}\left(\frac{1}{\sqrt{\delta^{2}-a x^{2}-b y^{2}}}\right) \mathrm{d} x \mathrm{~d} y
\end{aligned}
$$

Similar with Equation (4), we have Equation (5) is

$$
\frac{\delta^{2}}{\sqrt{c}} \iint_{a x^{2}+b y^{2} \leq \delta^{2}} \frac{1}{\sqrt{\delta^{2}-a x^{2}-b y^{2}}} \mathrm{~d} x \mathrm{~d} y=\frac{2 \pi}{\sqrt{a b c}}
$$

Then we obtain:

$$
\oiint_{\Sigma_{1}} \frac{x \mathrm{~d} y \mathrm{~d} z+y \mathrm{~d} z \mathrm{~d} x+z \mathrm{~d} x \mathrm{~d} y}{\left(a x^{2}+b y^{2}+c z^{2}\right)^{\frac{3}{2}}}=\frac{4 \pi}{\sqrt{a b c}}
$$

b) Take $\Sigma_{1}$ as a small cube surface $(x= \pm \sigma ; y= \pm \sigma ; z= \pm \sigma)$, see Figure 3 . Let:

$$
\pi_{1}: x=\delta,|y| \leq \delta,|z| \leq \delta \quad \pi_{2}: x=-\delta,|y| \leq \delta,|z| \leq \delta
$$
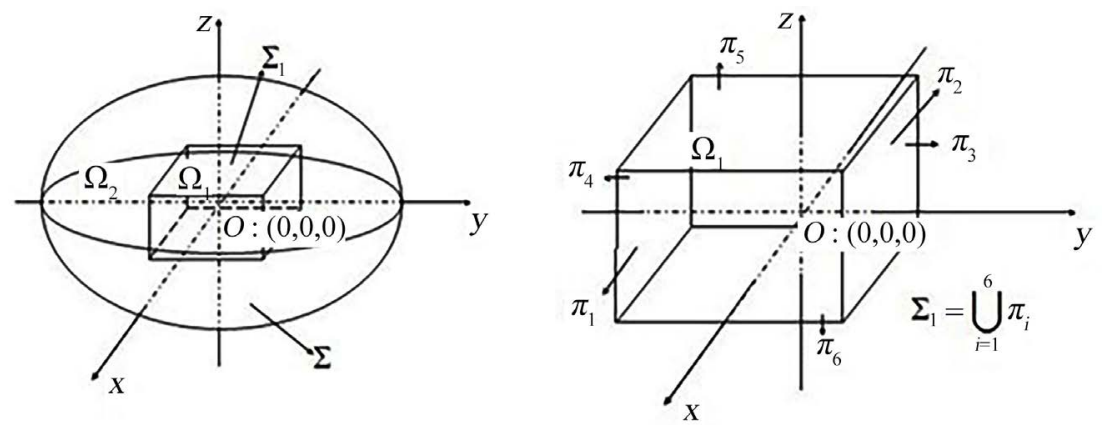

Figure 3. Graphs of $\Sigma$ and $\Sigma_{1}$. 


$$
\begin{gathered}
\pi_{3}: y=\delta,|x| \leq \delta,|z| \leq \delta \quad \pi_{4}: y=-\delta,|x| \leq \delta,|z| \leq \delta \\
\pi_{5}: z=\delta,|x| \leq \delta,|y| \leq \delta \quad \pi_{6}: z=-\delta,|x| \leq \delta,|y| \leq \delta \\
\Sigma_{1}=\bigcup_{i=1}^{6} \pi_{i}
\end{gathered}
$$

Then:

$$
\begin{aligned}
& \oiint_{\Sigma_{1}} \frac{x \mathrm{~d} y \mathrm{~d} z+y \mathrm{~d} z \mathrm{~d} x+z \mathrm{~d} x \mathrm{~d} y}{\left(a x^{2}+b y^{2}+c z^{2}\right)^{\frac{3}{2}}} \\
& =\iint_{\pi_{1} \cup \pi_{2}} \frac{x \mathrm{~d} y \mathrm{~d} z}{\left(a x^{2}+b y^{2}+c z^{2}\right)^{\frac{3}{2}}}+\iint_{\pi_{3} \cup \pi_{4}} \frac{y \mathrm{~d} z \mathrm{~d} x}{\left(a x^{2}+b y^{2}+c z^{2}\right)^{\frac{3}{2}}}+\iint_{\pi_{5} \cup \pi_{6}} \frac{z \mathrm{~d} x \mathrm{~d} y}{\left(a x^{2}+b y^{2}+c z^{2}\right)^{\frac{3}{2}}} \\
& =\iint_{\substack{y \leq \delta \delta \\
z \mid \leq \delta}} \frac{2 \delta \mathrm{d} y \mathrm{~d} z}{\left(a \delta^{2}+b y^{2}+c z^{2}\right)^{\frac{3}{2}}}+\iint_{\substack{x \leq \delta \leq \delta \\
|x| \leq \delta}} \frac{2 \delta \mathrm{d} z \mathrm{~d} x}{\left(a x^{2}+b \delta^{2}+c z^{2}\right)^{\frac{3}{2}}}+\iint_{\substack{|x| \leq \delta \leq \delta \\
y \leq \delta}} \frac{2 \delta \mathrm{d} x \mathrm{~d} y}{\left(a x^{2}+b y^{2}+c \delta^{2}\right)^{\frac{3}{2}}} \\
& =8 \delta\left[I_{1}+I_{2}+I_{3}\right]
\end{aligned}
$$

where:

$$
\begin{aligned}
& I_{1}=\iint_{\substack{0 \leq y \leq \delta \\
0 \leq x \leq \delta}} \frac{\mathrm{d} y \mathrm{~d} z}{\left(a \delta^{2}+b y^{2}+c z^{2}\right)^{\frac{3}{2}}} \\
& I_{2}=\iint_{\substack{0 \leq z \leq \delta \\
0 \leq x \leq \delta}} \frac{\mathrm{d} z \mathrm{~d} x}{\left(a x^{2}+b \delta^{2}+c z^{2}\right)^{\frac{3}{2}}} \\
& I_{3}=\iint_{\substack{0 \leq x \leq \delta \\
0 \leq y \leq \delta}} \frac{\mathrm{d} x \mathrm{~d} y}{\left(a x^{2}+b y^{2}+c \delta^{2}\right)^{\frac{3}{2}}}
\end{aligned}
$$

However, we can compute $I_{3}$ as follows:

$$
I_{3}=\iint_{\substack{0 \leq x \leq \delta \\ 0 \leq y \leq \delta}} \frac{\mathrm{d} x \mathrm{~d} y}{\left(a x^{2}+b y^{2}+c \delta^{2}\right)^{\frac{3}{2}}}=\int_{0}^{\delta} \int_{0}^{\delta} \frac{1}{\frac{3}{a^{2}}\left(x^{2}+\frac{b y^{2}+c \delta^{2}}{a}\right)^{\frac{3}{2}}} \mathrm{~d} x \mathrm{~d} y
$$

Using a simple equation:

$$
\int \frac{1}{\left(m^{2}+x^{2}\right)^{\frac{3}{2}}} \mathrm{~d} x=\frac{1}{m^{2}} \frac{x}{\left(m^{2}+x^{2}\right)^{\frac{1}{2}}}
$$

We have the follows:

$$
\begin{aligned}
& \int_{0}^{\delta} \int_{0}^{\delta} \frac{1}{a^{\frac{3}{2}}\left(x^{2}+\frac{b y^{2}+c \delta^{2}}{a}\right)^{\frac{3}{2}}} \mathrm{~d} x \mathrm{~d} y \\
& =\delta \int_{0}^{\delta} \frac{1}{\left(b y^{2}+c \delta^{2}\right) \sqrt{a \delta^{2}+c \delta^{2}+b y^{2}}} \mathrm{~d} y \\
& =\frac{\delta}{b^{\frac{3}{2}}} \int_{0}^{\delta} \frac{1}{\left(y^{2}+\frac{c}{b} \delta^{2}\right) \sqrt{y^{2}+\frac{a \delta^{2}+c \delta^{2}}{b}}} \mathrm{~d} y
\end{aligned}
$$


The key idea in solving Equation (7) is using proper variable transform, make sure the computation of Equation (7) is easier to come out.

Suppose:

$$
y=\sqrt{\frac{a \delta^{2}+c \delta^{2}}{b}} \tan \alpha
$$

Then:

$$
\begin{gathered}
\mathrm{d} y=\sqrt{\frac{a \delta^{2}+c \delta^{2}}{b}} \frac{1}{\cos ^{2} \alpha} \mathrm{d} \alpha \\
y^{2}+\frac{c}{b} \delta^{2}=\frac{a}{b} \delta^{2} \frac{\sin ^{2} \alpha}{\cos ^{2} \alpha}+\frac{c}{b \cos ^{2} \alpha} \delta^{2} \\
\sqrt{y^{2}+\frac{a \delta^{2}+c \delta^{2}}{b}}=\sqrt{\frac{a \delta^{2}+c \delta^{2}}{b}} \frac{1}{\cos \alpha}
\end{gathered}
$$

Meanwhile, the integration interval is known to be:

$$
0 \leq y=\sqrt{\frac{a \delta^{2}+c \delta^{2}}{b}} \tan \alpha \leq \delta
$$

This implies:

$$
\begin{gathered}
0 \leq \tan \alpha \leq \delta \sqrt{\frac{b}{a \delta^{2}+c \delta^{2}}}=\sqrt{\frac{b}{a+c}} \\
0 \leq \alpha \leq \arctan \sqrt{\frac{b}{a+c}}
\end{gathered}
$$

From Equation (8) and Equation (9), we have Equation (7) as:

$$
\begin{aligned}
& \frac{\delta}{b^{\frac{3}{2}}} \int_{0}^{\delta} \frac{\mathrm{d} y}{\left(y^{2}+\frac{c}{b} \delta^{2}\right) \sqrt{y^{2}+\frac{a \delta^{2}+c \delta^{2}}{b}}} \\
& =\frac{\delta}{b^{\frac{3}{2}}} \int_{0}^{\arctan \sqrt{\frac{b}{a+c}}} \frac{\sqrt{\frac{a \delta^{2}+c \delta^{2}}{b}} \frac{1}{\cos ^{2} \alpha} \mathrm{d} \alpha}{\left(\frac{a}{b} \delta^{2} \frac{\sin ^{2} \alpha}{\cos ^{2} \alpha}+\frac{c}{b \cos ^{2} \alpha} \delta^{2}\right) \sqrt{\frac{a \delta^{2}+c \delta^{2}}{b}} \frac{1}{\cos \alpha}} \\
& =\frac{1}{\delta b^{\frac{1}{2}}} \int_{0}^{\arctan \sqrt{\frac{b}{a+c}}} \frac{\cos \alpha}{a \sin ^{2} \alpha+c} \mathrm{~d} \alpha
\end{aligned}
$$

Suppose:

$$
x=\sin \alpha
$$

Then we show that:

$$
\begin{array}{r}
0 \leq \tan \alpha \leq \sqrt{\frac{b}{a+c}} \\
0 \leq \frac{\sin \alpha}{\sqrt{1-\sin ^{2} \alpha}} \leq \sqrt{\frac{b}{a+c}}
\end{array}
$$




$$
\sin \alpha \geq 0 \text { and } \sin ^{2} \alpha \leq \frac{b}{a+b+c}
$$

From Equation (11) Equation (12), we have Equation (10) as:

$$
\frac{1}{\delta b^{\frac{1}{2}}} \int_{0}^{\arctan \sqrt{\frac{b}{a+c}}} \frac{\cos \alpha}{a \sin ^{2} \alpha+c} \mathrm{~d} \alpha=\frac{1}{a \delta b^{\frac{1}{2}}} \int_{0}^{\sqrt{\frac{b}{a+b+c}}} \frac{1}{x^{2}+\frac{c}{a}} \mathrm{~d} x
$$

Apply $\int \frac{1}{m^{2}+x^{2}} \mathrm{~d} x=\frac{1}{m} \arctan \frac{x}{m}+C$ to Equation (13) to obtain:

$$
\begin{aligned}
& \frac{1}{a \delta b^{\frac{1}{2}}} \int_{0}^{\sqrt{\frac{b}{a+b+c}}} \frac{1}{x^{2}+\frac{c}{a}} \mathrm{~d} x \\
& =\frac{1}{\sqrt{a b c} \delta}\left(\arctan \sqrt{\frac{a b}{c a+c b+c^{2}}}-\arctan 0\right) \\
& =\frac{1}{\sqrt{a b c} \delta} \arctan \sqrt{\frac{a b}{c a+c b+c^{2}}}
\end{aligned}
$$

Similarly:

$$
\begin{aligned}
& I_{1}=\frac{1}{\sqrt{a b c} \delta} \arctan \sqrt{\frac{b c}{a b+a c+a^{2}}} \\
& I_{2}=\frac{1}{\sqrt{a b c} \delta} \arctan \sqrt{\frac{a c}{a b+b c+b^{2}}}
\end{aligned}
$$

Then:

$$
\begin{aligned}
\oiint_{\Sigma_{1}} \frac{x \mathrm{~d} y \mathrm{~d} z+y \mathrm{~d} z \mathrm{~d} x+z \mathrm{~d} x \mathrm{~d} y}{\left(x^{2}+y^{2}+z^{2}\right)^{\frac{3}{2}}} \\
=8 \delta\left[I_{1}+I_{2}+I_{3}\right] \\
=\frac{8}{\sqrt{a b c}}\left(\arctan \sqrt{\frac{b c}{a b+a c+a^{2}}}+\arctan \sqrt{\frac{a c}{a b+b c+b^{2}}}\right) \\
+\frac{8}{\sqrt{a b c}}\left(\arctan \sqrt{\frac{a b}{c a+c b+c^{2}}}\right)
\end{aligned}
$$

Let $\tan A=\sqrt{\frac{b c}{a b+a c+a^{2}}} ; \tan B=\sqrt{\frac{a c}{a b+b c+b^{2}}} ; \tan C=\sqrt{\frac{a b}{c a+c b+c^{2}}}$

Next we show that the sum of $A+B+C$ is $\frac{\pi}{2}$.

With:

$$
\tan [(A+B)+C]=\frac{\tan (A+B)+\tan C}{1-\tan (A+B) \tan C}=\frac{\frac{\tan A+\tan B}{1-\tan A \cdot \tan B}+\tan C}{1-\frac{\tan A+\tan B}{1-\tan A \cdot \tan B} \tan C}
$$

Therefore, the denominator of Equation (15) is: 


$$
1-\frac{\tan A+\tan B}{1-\tan A \cdot \tan B} \tan C=1-\frac{\frac{b}{a+b+c}+\frac{a}{a+b+c}}{\frac{a+b}{a+b+c}}=0
$$

the numerator of Equation (15) is:

$$
\frac{\tan A+\tan B}{1-\tan A \cdot \tan B}+\tan C=\frac{\frac{1}{a} \sqrt{\frac{a b c}{a+b+c}}+\frac{1}{b} \sqrt{\frac{a b c}{a+b+c}}}{1-\frac{1}{a b} \cdot \frac{a b c}{a+b+c}}+\frac{1}{c} \sqrt{\frac{a b c}{a+b+c}} \neq 0
$$

Equations (15)-(17) imply the value of $A+B+C$ is $\frac{\pi}{2}$, in other words:

$$
\arctan \sqrt{\frac{b c}{a b+a c+a^{2}}}+\arctan \sqrt{\frac{a c}{a b+b c+b^{2}}}+\arctan \sqrt{\frac{a b}{c a+c b+c^{2}}}=\frac{\pi}{2}
$$

From Equation (18) we have Equation (15) as follows:

$$
\begin{aligned}
& \frac{8}{\sqrt{a b c}}\left(\arctan \sqrt{\frac{b c}{a b+a c+a^{2}}}+\arctan \sqrt{\frac{a c}{a b+b c+b^{2}}}+\arctan \sqrt{\frac{a b}{c a+c b+c^{2}}}\right) \\
& =\frac{8}{\sqrt{a b c}} \times \frac{\pi}{2}=\frac{4 \pi}{\sqrt{a b c}}
\end{aligned}
$$

Then we obtain:

$$
\oiint_{\Sigma_{1}} \frac{x \mathrm{~d} y \mathrm{~d} z+y \mathrm{~d} z \mathrm{~d} x+z \mathrm{~d} x \mathrm{~d} y}{\left(a x^{2}+b y^{2}+c z^{2}\right)^{\frac{3}{2}}}=\frac{4 \pi}{\sqrt{a b c}}
$$

Remark 2: 1) The result of Equation (18) is proved by computer simulation, the process is given in the appendix.

2) Lemma 1 is a generalization of the Gauss theorem. Theorem 1 is a general expression for the lemma 1.

3) From theorem 1, it is sufficient to compute the surface integrals in vector fields, such as Example 1 and Example 2.

Example 1:

$$
\oiint_{\Sigma} \frac{x \mathrm{~d} y \mathrm{~d} z+y \mathrm{~d} z \mathrm{~d} x+z \mathrm{~d} x \mathrm{~d} y}{\left(x^{2}+y^{2}+z^{2}\right)^{\frac{3}{2}}}=4 \pi
$$

Example 2:

$$
\oiint_{\Sigma} \frac{x \mathrm{~d} y \mathrm{~d} z+y \mathrm{~d} z \mathrm{~d} x+z \mathrm{~d} x \mathrm{~d} y}{\left(x^{2}+y^{2}+z^{2}\right)^{\frac{3}{2}}}=2 \pi
$$

Lots of surface integrals are integrals of the form $\oiint_{\Sigma} \frac{x \mathrm{~d} y \mathrm{~d} z+y \mathrm{~d} z \mathrm{~d} x+z \mathrm{~d} x \mathrm{~d} y}{\left(a x^{2}+b y^{2}+c z^{2}\right)^{\frac{3}{2}}}$.

\section{Funding}

Natural Science Foundation of Chongqing (Grant No. cstc2020jcyj-msxmX0232); Higher Education Research Project of Chongqing Municipal (Grant No. 203332). 


\section{Conflicts of Interest}

The authors declare no conflicts of interest.

\section{References}

[1] Michiel, H. (ed.) (2001) Surface Integral, Encyclopedia of Mathematics. Springer, Heidelberg.

[2] Bronshtein, I.N. and Semendyayev, K.A. (2007) Handbook of Mathematics. 5th Edition. Springer, Heidelberg.

[3] Wilfred, K. (2002) Advanced Calculus. Jones and Bartlett Publishers, Burlington.

\section{Appendix}

Suppose $a, b, c$ as follows:

1). $a=1, b=1, c=12) . a=1, b=2, c=3$

3). $a=1, b=2, c=4 \quad 4) . a=1, b=3, c=5$

5). $a=2, b=3, c=5$ 6). $a=3, b=4, c=5$

According to Equation (18), using R we can obtain:

$>3.1415926=2$

[1] 1.570796

$>\operatorname{atan}\left((1 / 3)^{\wedge}(1 / 2)\right)+\operatorname{atan}\left((1 / 3)^{\wedge}(1 / 2)\right)+\operatorname{atan}\left((1 / 3)^{\wedge}(1 / 2)\right)$

[1] 1.570796

$>\operatorname{atan}\left((1 / 1)^{\wedge}(1 / 2)\right)+\operatorname{atan}\left((1 / 4)^{\wedge}(1 / 2)\right)+\operatorname{atan}\left((1 / 9)^{\wedge}(1 / 2)\right)$

[1] 1.570796

$>\operatorname{atan}\left((8 / 7)^{\wedge}(1 / 2)\right)+\operatorname{atan}\left(\left(8 /\left(4^{\star} 7\right)\right)^{\wedge}(1 / 2)\right)+\operatorname{atan}\left(\left(8 /\left(16^{\star} 7\right)\right)^{\wedge}(1 / 2)\right)$

[1] 1.570796

$>\operatorname{atan}\left(\left(24 /\left(4^{*} 9\right)\right)^{\wedge}(1 / 2)\right)+\operatorname{atan}\left(\left(24 /\left(9^{*} 9\right)\right)^{\wedge}(1 / 2)\right)+\operatorname{atan}\left(\left(24 /\left(16^{*} 9\right)\right)^{\wedge}(1 / 2)\right)$

[1] 1.570796

$>\operatorname{atan}\left(\left(30 /\left(4^{\star} 10\right)\right)^{\wedge}(1 / 2)\right)+\operatorname{atan}\left(\left(30 /\left(9^{\star} 10\right)\right)^{\wedge}(1 / 2)\right)+\operatorname{atan}\left(\left(30 /\left(25^{\star} 10\right)\right)^{\wedge}(1 / 2)\right)$

[1] 1.570796

$>\operatorname{atan}\left((5 / 9)^{\wedge}(1 / 2)\right)+\operatorname{atan}\left((5 / 16)^{\wedge}(1 / 2)\right)+\operatorname{atan}((5 / 25) \wedge(1 / 2))$

[1] 1.570796 